\title{
Phase transformations induced in relaxed amorphous silicon by indentation at room temperature
}

\author{
B. Haberl, ${ }^{a}$ J. E. Bradby, ${ }^{\text {b) }}$ M. V. Swain, ${ }^{\text {c) }}$ and J. S. Williams \\ Department of Electronic Materials Engineering, Research School of Physical Sciences and Engineering, \\ The Australian National University, Canberra, ACT 0200, Australia \\ P. Munroe \\ Electron Microscope Unit, University of New South Wales, Sydney, NSW 2052, Australia
}

(Received 7 June 2004; accepted 15 October 2004)

\begin{abstract}
The deformation behavior of self-ion-implanted amorphous-Si $(a-\mathrm{Si})$ has been studied using spherical nanoindentation in both relaxed (annealed) and unrelaxed (as-implanted) $a$-Si. Interestingly, phase transformations were clearly observed in the relaxed state, with the load-unload curves from these samples displaying characteristic discontinuities and cross-sectional transmission electron microscopy images indicating the presence of high-pressure crystalline phases Si-III and $\mathrm{Si}$-XII following pressure release. Thus, an amorphous to crystalline phase transformation has been induced by indentation at room temperature. In contrast, no evidence of a phase transformation was observed in unrelaxed $a$-Si, which appeared to deform via plastic flow of the amorphous phase. Furthermore, in situ electrical measurements clearly indicate the presence of a metallic Si phase during loading of relaxed $a$-Si but no such behavior was observed for unrelaxed $a$-Si (c) 2004 American Institute of Physics. [DOI: 10.1063/1.1832757]
\end{abstract}

Crystalline silicon $(c-\mathrm{Si})$ is well known to undergo a series of phase transformations during mechanical deformation. ${ }^{1-6}$ From both high-pressure diamond anvil and indentation experiments, it has been shown that crystalline diamond-cubic Si-I undergoes a phase transformation to a metallic $\beta$-Sn (Si-II) phase during loading at a pressure of $\sim 11 \mathrm{GPa}{ }^{1,2} \mathrm{As} \mathrm{Si}-\mathrm{II}$ is unstable at pressures below $2 \mathrm{GPa}$, the material undergoes further transformation during pressure release. The final structure of the transformed volume after indentation has been found to depend critically on the rate of pressure release, with slow unloading rates leading to the formation of polycrystalline phases (Si-III and Si-XII) and fast pressure release rates resulting in the transformation to an amorphous silicon $\left(a\right.$-Si) phase. ${ }^{4}$

In this letter we examine the indentation behavior in $a-\mathrm{Si}$, which is an interesting material in its own right. Indeed, isostatic diamond-anvil compression of this phase of Si suggests that it undergoes a transformation to a high-density (amorphous) metallic phase on loading and transforms back to the original low-density amorphous phase on unloading. ${ }^{7}$ That is, diamond-anvil experiments indicate that, whereas $c$-Si transforms to a number of high-pressure crystalline phases on loading, $a$-Si transforms to a higher density amorphous phase and remains amorphous throughout the loading/ unloading cycle. Furthermore, early indentation measurements on ion-implanted $a$-Si showed that the mechanical response appeared to depend on the preparation conditions or the "state" of the amorphous phase. ${ }^{8}$ The so-called relaxed $a$-Si $\operatorname{state}^{9}$ (in which the implanted $a$-Si layer was annealed to $\sim 450{ }^{\circ} \mathrm{C}$ prior to indentation) exhibited very similar loadunload indentation curves to those of $c$-Si, including a similar hardness. On the other hand, unannealed (unrelaxed) $a-\mathrm{Si}$

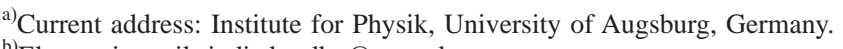

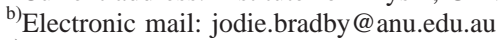

${ }^{c}$ Visiting Fellow at The Australian National University. Present address:

Oral Science Department, Faculty of Dentistry, Dunedin, New Zealand.
}

appeared to deform plastically at lower loads and exhibit a somewhat lower hardness. More recently, indentation measurements of Follstaedt et al. ${ }^{10}$ confirmed that the hardness of relaxed $a$-Si approached that of $c$-Si but that unrelaxed $a$-Si was slightly softer. These authors inferred that the overall similarity of hardness values between crystalline and both states of $a$-Si suggested that phase transformations occur during loading in all cases. However, no direct evidence has been given in previous indentation studies of $a$-Si to confirm that a phase transformation had actually occurred.

Finally, the previous diamond-anvil and indentation measurements raise some important general questions that we address in this letter. Does $a$-Si undergo a phase transformation under indentation loading, and, if so, does it transform to a high-density (amorphous) metallic phase on loading and back to the low-density amorphous phase on unloading? Furthermore, do the two states of low-density $a$-Si (relaxed and unrelaxed) deform by similar processes?

In this current study a continuous layer of $a$-Si was prepared by ion-implantation of $c$-Si with $400 \mathrm{keV} \mathrm{Si}$ ions at liquid nitrogen temperature using the ANU 1.7 MV tandem accelerator. After implantation, a portion of the sample was annealed for $30 \mathrm{~min}$ at a temperature of $\sim 450{ }^{\circ} \mathrm{C}$ in an argon atmosphere to fully relax ${ }^{11}$ the $a$-Si layer but not sufficient to induce any significant crystallization. The thickness of both the relaxed (annealed) and unrelaxed (unannealed) $a$-Si layers were measured to be around $600-650 \mathrm{~nm}$ by Rutherford backscattering with $2 \mathrm{MeV}$ helium ions. A series of indentations were then performed on both unrelaxed and relaxed $a$-Si samples, as well as on a completely crystalline $\mathrm{Si}$ sample for comparison. The indentations were made using an Ultra-Micro-Indentation-System 2000 with a spherical indenter of $\sim 2.0 \mu \mathrm{m}$ radius, at room temperature and pressure. Loads of up to $20 \mathrm{mN}$ were applied (penetrating up to approximately one-third of the amorphous layer) at loading and unloading rates of $\sim 1-2 \mathrm{mN} / \mathrm{s}$ using a continuous loadunload cycle. After indentation, the residual indent impres- 

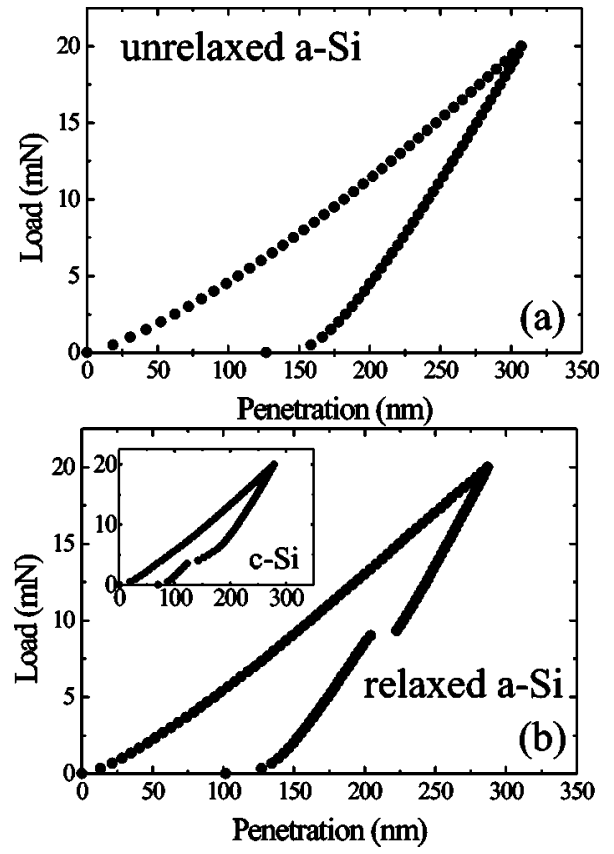

FIG. 1. Load-unload curves of (a) unrelaxed and (b) relaxed $a$-Si loaded to $20 \mathrm{mN}$ using a continuous load cycle with a spherical indenter of $\sim 2 \mu \mathrm{m}$ radius. Inset to (b) shows $c$-Si indented using the same conditions.

sions were prepared for cross-sectional transmission electron microscopy (XTEM) using a FEIxP200 focused ion beam (FIB) system. Transmission electron microscopy (TEM) was carried out in a Philips CM 300 operating at an accelerating voltage of $300 \mathrm{kV}$. In situ electrical measurements ${ }^{12}$ were carried out during indentation using $a$-Si layers that were thin enough $(\sim 100 \mathrm{~nm})$ to allow current to be measured through the sample during indentation if transformation to a metallic Si phase occurred.

Figure 1(a) shows a load versus penetration plot of unrelaxed $a$-Si loaded to $20 \mathrm{mN}$ using a spherical indenter with a radius of $\sim 2.0 \mu \mathrm{m}$. No discontinuities on either the loading or unloading sections of the curve are observed. In contrast, the nanoindentation curve taken from relaxed $a-\mathrm{Si}$, as shown in Fig. 1(b), exhibits a clear discontinuity on unloading. Such an event is typically observed when indenting $c-\mathrm{Si}$ [in an inset to Fig. 1(b)] and is referred to as a "pop-out" event. ${ }^{6,13}$ Furthermore, $c$-Si is well known to exhibit a discontinuity on loading with a spherical indenter (called "popin") but such a discontinuity is not as easily observed when indenting relaxed $a$-Si. Indeed, such discontinuities during indentation have been reported to occur for $c$-Si as a result of pressure-induced phase transformations. ${ }^{6,14}$ Figure 1 also shows that the maximum depth of penetration is $\sim 30 \mathrm{~nm}$ greater for the unrelaxed $a-\mathrm{Si}$ compared to either relaxed $a$-Si or $c$-Si. This is consistent with previous studies ${ }^{8,10}$ which have observed that unrelaxed $a$-Si is measurably softer than relaxed $a$-Si or $c$-Si.

Figure 2(a) shows a XTEM bright-field image of a 20 $\mathrm{mN}$ indent in unrelaxed $a$-Si made using a $\sim 2 \mu \mathrm{m}$ radius indenter. A selected area diffraction pattern (SADP) of the region immediately beneath the residual indent (shown as an inset to this figure) shows only the diffuse rings characteristic an amorphous structure. In contrast, the dark-field (DF) image of a residual indent in relaxed $a-\mathrm{Si}$, shown in Fig. 2(b), illustrates very clearly that phase transformations have occurred during loading and unloading. The DF image was phases on unloading.
Downloaded 10 Jan 2005 to 150.203 .178 .66 . Redistribution subject to AIP license or copyright, see http://apl.aip.org/apl/copyright.jsp
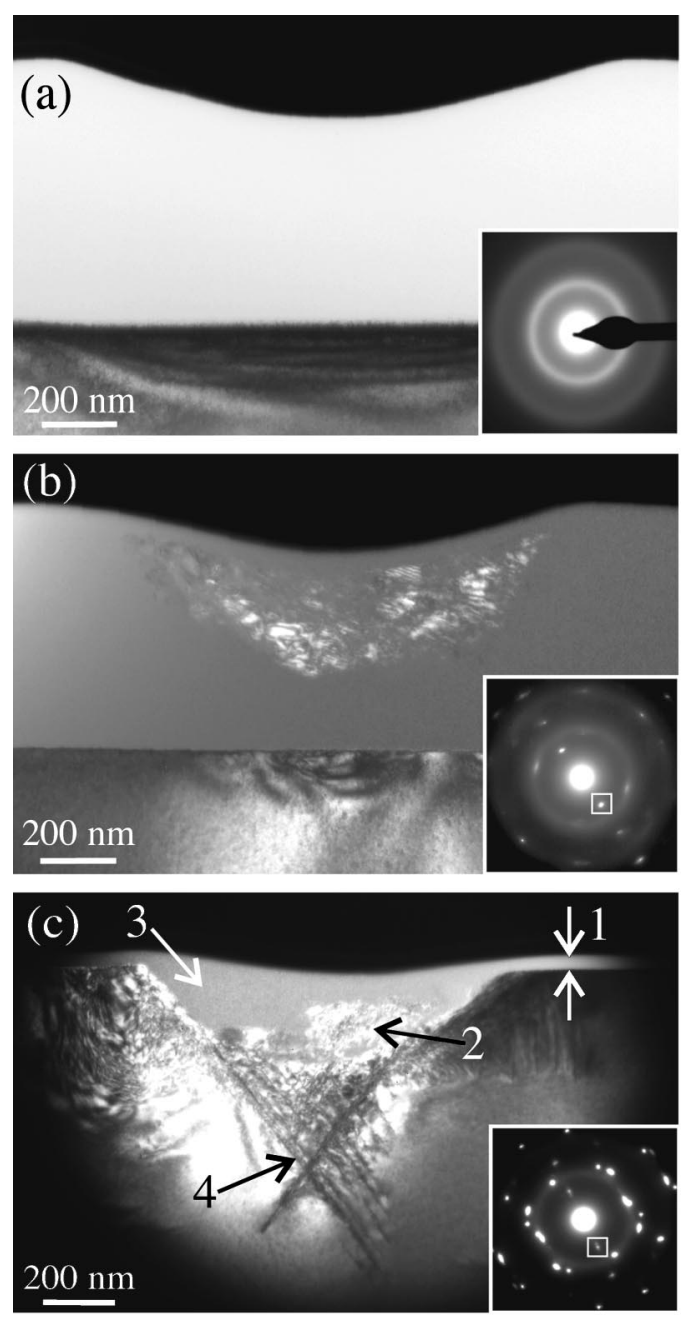

FIG. 2. (a) Bright-field XTEM image of an indent in unrelaxed amorphous silicon; (b) DF XTEM image of an indent in relaxed amorphous silicon, the diffraction spot used to form the DF image is marked with a box in the inset; and (c) DF XTEM image of an indent in $c$-Si taken using the boxed diffraction spot. Region 1 is the $a$-Si layer due to FIB milling, region 2 denotes the $\mathrm{Si}-\mathrm{XII} / \mathrm{Si}$-III phases, region 3 shows $a-\mathrm{Si}$, and region 4 shows slip. All indents were made using a continuous load cycle with a $\sim 2 \mu \mathrm{m}$ radius indenter to a maximum load of $20 \mathrm{mN}$. The SADP were taken from the regions directly under the residual indent impressions.

taken using the boxed diffraction spot from $\mathrm{Si}$-III/Si-XII shown in the inset to this figure. These extra diffraction spots are consistent with results from previous work ${ }^{5}$ as arising from Si-III/XII. No slip or transformation was observed to occur in the underlaying $c$-Si substrate. In situ electrical measurements confirmed that an electrically conducting (metallic) layer had formed during loading for the relaxed $a-\mathrm{Si}$ case and this layer further transformed on unloading to a less-conductive phase. For unrelaxed $a-\mathrm{Si}$, no conduction was measured at any stage of the loading or unloading cycle.

For comparison with the behavior in $a-\mathrm{Si}$, a DF image of an indent in $c$-Si loaded under identical indentation conditions is shown in Fig. 2(c). Consistent with previous observations, phase transformations to both $a$-Si and the polycrystalline $\mathrm{Si}-\mathrm{XII} / \mathrm{Si}$-III phases occur during unloading, as well as slip resulting from shear-stress-induced deformation prior to transformation. ${ }^{5}$ Furthermore, as has also been shown previously in $c-\mathrm{Si}^{12}{ }^{12}$ in situ electrical measurements are able to monitor the formation of the conducting (metallic) phase on loading and subsequent transformation to less conducting phases on unloading.

AIP license or copyright, see http://apl.aip.org/apl/copyright.jsp 
The above-mentioned results clearly show that it is possible to induce phase transformations in relaxed $a$-Si at room temperature by simple mechanical deformation. Furthermore, we show that crystalline phases (Si-III/Si-XII) can result following indentation. This result is intriguing in two respects: (i) it constitutes direct evidence that room temperature indentation of $\mathrm{Si}$ can cause transformation from an amorphous phase to a crystalline phase; and (ii) it appears to contradict behavior previously observed during diamondanvil (isostatic) loading experiments where no crystalline phases were observed at any stage, an issue we discuss later in this letter.

There has been much interest over the past two decades in the structure and other property differences between relaxed and unrelaxed $a$-Si. ${ }^{9-11,15-19}$ Whereas these states of $a$-Si appear identical to measurements sensitive to short range order, such as details of the radial distribution function obtained from TEM, ${ }^{15}$ other measurements such as $\mathrm{IR}^{17}$ and Raman spectroscopy spectra, ${ }^{18}$ calorimetry measurements ${ }^{11}$ and, more recently, fluctuation electron microscopy ${ }^{16}$ can identify structural differences between them. Indeed, in terms of mechanical deformation of $a-\mathrm{Si}$, such attempts to explain the differences between relaxed and unrelaxed $a-\mathrm{Si}$ can provide some basis for explaining the softer behavior of unrelaxed $a-\mathrm{Si}$, that deforms via plastic flow, compared with phase transformations occurring in relaxed $a$-Si. For example, a high defect or dangling bond density suggested by previous studies of unrelaxed $a-\mathrm{Si}^{9,11,17}$ may aid material flow by facilitating propagation of defects or dangling bonds. Furthermore, detailed fluctuation microscopy measurements of Gibson et al. ${ }^{15,16}$ have led these authors to propose, somewhat controversially, that unrelaxed $a$-Si may contain medium-range ordered "paracrystals", which we suggest could also similarly favor plastic deformation via "grain boundary" shear.

In relation to relaxed $a$-Si, calorimetry measurements suggest that a decrease in the average bond angle distortion during annealing may be the dominant mechanism involved in structural relaxation ${ }^{11}$ and all previous studies ${ }^{9,11,15-19}$ indicate that the relaxed state is a continuous random network (CRN). Hence, such a CRN with almost full coordination (low dangling bond density) may give rise to mechanical behavior and deformation properties that are very similar to $c$-Si.

It is also important to comment on the possible role of hydrogen in $a-\mathrm{Si}$ in terms of its effect on mechanical behavior under our preparation conditions. In our case, annealed $a$-Si might be expected to contain a hydrogen concentration around $1 \times 10^{19} \mathrm{~cm}^{-3}$, whereas as-implanted $a$-Si would simply contain hydrogen at a concentration below the solubility limit in $c$-Si $\left(\sim 1 \times 10^{12} \mathrm{~cm}^{-3}\right)$. The reason for this is that hydrogen trapped within the native oxide and in the annealing ambient is known to diffuse into $a$-Si during annealing. ${ }^{19}$ Thus, it could be argued that it is the presence of a much higher hydrogen content in relaxed $a$-Si that controls its ability to transform in comparison with unrelaxed $a-\mathrm{Si}$. To fully resolve this issue it will be necessary to carry out further experiments.

Let us now address the observed difference between the diamond-anvil experiments and our indentation results. First, $a$-Si films that are vapor-deposited under less than ultrahigh vacuum conditions (as in the previous diamond-anvil experiments) will both contain a higher concentration of impurities than $a$-Si made by ion-implantation and also exhibit a higher porosity (lower density) than that of implanted $a-\mathrm{Si}^{20} \mathrm{We}$ suggest that impurities and a porous microstructure may therefore stabilize and preserve the amorphous phase during load/unloading of diamond-anvil experiments. In our case, pure, relaxed $a-\mathrm{Si}$ may transform to the lowest free-energy crystalline phases during pressure-induced deformation. Second, the uniform, pure isostatic pressure that characterizes diamond-anvil experiments compared with highly nonuniform pressure and an appreciable shear component in the case of indentation, may lead to differences in mechanical deformation of $a$-Si. Indeed, for loading of $c$-Si, no amorphous phase is found after diamond-anvil experiments whereas fast unloading during indentation favors the formation of $a$-Si. Finally, the state of the $a$-Si in the diamondanvil case is almost certainly unrelaxed, which is the usual state of deposited $a-\mathrm{Si}^{9}{ }^{9}$ whereas it is only our relaxed $a-\mathrm{Si}$ that undergoes phase transformation during loading. Hence, the degree of relaxation of the starting $a$-Si may be the dominant determinant of the mode of mechanical deformation (plastic flow versus phase transformation).

In conclusion we have observed an amorphous to crystalline phase transformation at room temperature in relaxed $a$-Si resulting in the end phases $\mathrm{Si}-\mathrm{III}$ and $\mathrm{Si}$-XII. A clear difference has also been observed between the deformation behavior of relaxed and unrelaxed $a-\mathrm{Si}$, with no phase transformations being observed in the latter case. These results are significant and warrant further investigation to fully understand the specific kinetic and thermodynamic pathways to phase transformation under mechanical deformation of $a$-Si.

${ }^{1}$ J. Z. Hu, L. D. Merkle, C. S. Menoni, and I. L. Spain, Phys. Rev. B 34, 4679 (1986).

${ }^{2}$ R. O. Piltz, J. R. Maclean, S. J. Clark, G. J. Ackland, P. D. Hatton, and J. Crain, Phys. Rev. B 52, 4072 (1995).

${ }^{3}$ D. R. Clarke, M. C. Kroll, P. D. Kirchner, R. F. Cook, and B. J. Hockey, Phys. Rev. Lett. 60, 2156 (1988)

${ }^{4}$ V. Domnich, Y. Gogotsi, and S. Dub, Appl. Phys. Lett. 76, 2214 (2000).

${ }^{5}$ J. E. Bradby, J. S. Williams, J. Wong-Leung, M. V. Swain, and P. Munroe, Appl. Phys. Lett. 77, 3749 (2000).

${ }^{6}$ J. E. Bradby, J. S. Williams, J. Wong-Leung, M. V. Swain, and P. Munroe, J. Mater. Res. 16, 1500 (2001).

${ }^{7}$ S. K. Deb, M. Wilding, M. Somayazulu, and P. F. McMillian, Nature (London) 414, 528 (2001).

${ }^{8}$ J. S. Williams, J. S. Field, and M. V. Swain, Mater. Res. Soc. Symp. Proc. 308, 571 (1993).

${ }^{9}$ S. Roorda, W. C. Sinke, J. M. Poate, D. C. Jacobson, S. Dieker, B. S. Dennis, D. J. Eaglesham, F. Spaepen, and P. Fuoss, Phys. Rev. B 44, 3702 (1991).

${ }^{10}$ D. M. Follstaedt, J. A. Knapp, and S. M. Myers, J. Mater. Res. 19, 338 (2004).

${ }^{11}$ S. Roorda, S. Doorn, W. C. Sinke, P. M. L. O. Scholte, and E. van Loenen, Phys. Rev. Lett. 62, 1880 (1989)..

${ }^{12}$ J. E. Bradby, J. Williams, and M. V. Swain, Phys. Rev. B 67, 085205 (2003).

${ }^{13}$ I. Zarudi, L. C. Zhang, and M. V. Swain, Appl. Phys. Lett. 82, 1027 (2003).

${ }^{14}$ Y. G. Gogotsi, V. Domnich, S. N. Dub, A. Kailer, and K. G. Nickel, J. Mater. Res. 15, 871 (2000).

${ }^{15}$ J. M. Gibson, J.-Y. Cheng, P. Voyles, M. M.J. Treacy, and D. C. Jacobson, Mater. Res. Soc. Symp. Proc. 540, 27 (1999).

${ }^{16}$ J. M. Gibson and M. M. J. Treacy, Phys. Rev. Lett. 78, 1074 (1997).

${ }^{17}$ J. E. Frederickson, C. N. Waddell, W. G. Spitzer, and G. K. Hubler, Appl. Phys. Lett. 40, 172 (1982)

${ }^{18}$ R. Tsu, J. Gonzalez-Hernandez, J. Doehler, and S. R. Ovshinsky, Solid State Commun. 46, 79 (1983)

${ }^{19}$ J. A. Roth, G. L. Olson, D. C. Jacobson, and J. M. Poate, Amorphous Silicon Technology - Symposium, Mater. Res. Soc., 1993, 291-296

${ }^{20}$ J. C. Bean and J. M. Poate, Appl. Phys. Lett. 36, 59 (1980). 